\title{
Effect of the Short-Segment Internal Fixation with Intermediate Inclined-Angle polyaxial Screw at the Fractured Vertebrae on the Treatment of Denis type B Thoracolumbar Fracture
}

\section{Chengjie Xiong}

PLA Central Military Command Region General Hospital

\section{Biwang Huang}

Wuhan University of Science and Technology

\section{Tanjun Wei}

General hospital of central theater command of PLA

\section{Hui Kang}

General Hospital of Central Theater Command of PLA

Feng Xu ( $\sim$ fengxu1969@163.com )

General Hospital of Central Theater Command of PLA

\section{Research article}

Keywords: Denis type B Thoracolumbar Fracture, Short-Segment Internal Fixation, Intermediate InclinedAngle Screw

Posted Date: March 18th, 2020

DOI: https://doi.org/10.21203/rs.2.22037/v2

License: (c) (i) This work is licensed under a Creative Commons Attribution 4.0 International License.

Read Full License

Version of Record: A version of this preprint was published at Journal of Orthopaedic Surgery and Research on May 24th, 2020. See the published version at https://doi.org/10.1186/s13018-020-01686-7. 


\section{Abstract}

Backgroud Short-segment internal fixation with straight-forward monoaxial screw (SSIF-SFM) and longsegment internal fixation (LSIF) are two major surgical options for thoracolumbar (TL) fracture, however, limitations of both surgical options I are obvious. SSIF with inclined-angle polyxial screw (SSIF-IAP) have been developed to take advantage of their benefits and minimize their adverse effects .

Methods 69 consecutive patients ( 47 males and 22 females; average 34.5 years ) who met the criteria for inclusion were enrolled in this study. Sagittal Cobb's angle (SCA), anterior vertebral body height (AVBH), vertebral body index (VBI) and spinal canal encroachment (SCE) were measured and assessed . Functional recovery Visual Analogue Scale (VAS) and Oswestry disability index (ODI) were also evaluated.

Results The value of incision length, mean blood loss, duration of operation and hospital stay in the SSIF-IAP group and SSIF-SFM group were significantly lower than those in the LSIF group. The AVBH and VBI in the SSIF-IA P group and LSIF group were significantly more improved than those in the SSIFSFM group at 6 months and the latest follow-ups $(p<0.05)$. The correction losses of AVBH and VBI ( calculated by the reduction of AVBH and VBI) in the SSIF-IAP group and LSIF group were also significantly lower than those in the SSIF-SFM group at 6 months and the latest follow-ups $(P<0.05)$. There was no significant difference of SCE among three groups. The VAS and ODI in the SSIF-IAP group and SSIF-SFM group were significantly lower than those in the LSIF group at 6 months and the latest follow-ups $(P<0.05)$.

Conclusion SSIF-IAP can achieve comparable treatment outcomes compared with LSIF, but it was less invasive compared with LSIF. The SSIF-IA was an effective and reliable operative technique for patients with Denis type B TL fracture.

\section{Introduction}

The thoracolumbar $(T L)$ junction is a transition zone between the rigid thoracic spine and the more mobile lumbar spine (from T11 to L2), and nearly $70 \%$ of all traumatic spinal injuries occur within this region [1, 2].The treatment of TL fracture remains under debate, especially in patients without serious neurological symptoms. Although conservative treatment seems to be effective in the majority of patients, clinical studies have shown that surgical treatment provides better fracture reduction, stronger fixation and more favorable long-term clinical outcomes $[3,4]$. The posterior approach for the surgical treatment of TL fracture is often preferred $[5,6]$. There are two main reasons for this choice. Firstly, more postoperative complications are associated with the anterior approach as compared with the posterior approach [7-9]. In addition, spine surgeons are more familiar with the posterior approach due to its easier application [10]. 
Short-segment internal fixation (SSIF) via posterior approach is the most common treatment for TL fracture[11]. Although SSIF can obtain satisfactory reduction, it often led to instrumentation failure due to osteoporosis and correction loss [12]. Long-segment internal fixation (LSIF) is an alternative solution, which increase construct stiffness and reduce the load on each screw by the application of longer segmental instrumentation, however, LSIF is unnecessarily extensive and decrease the number of motion segments. In addition, LSIF is often associated with the development of adjacent-segment degeneration (ASD) disease [13-15]. Saving the motion segments is the crucial principle of spinal surgery. Therefore, in order to restrict the number of fusion segments and improve instrumentation efficiency, additional intermediate screws inserted at the fracture level is applied along with the SSIF to treat TL fracture[16, 17]. The intermediate screws in this SSIF system are usually paralleled with the superior endplate with relatively short length[18]. However, as a result of collapse of anterior and middle columns in TL fracture, SSIF with straight-forward monoaxial screws can't always provide sufficient biomechanical support for the anterior column[19].

Recently, studies $[20,21]$ have shown that relatively long length pedicle screws could significantly increase the pullout force and the stiffness of the fixation system. We subsequently modified the traditional SSIF with straight-forward monoaxial screws and developed a new technique for TL fracture. We changed the direction of implementation of pedicle trajectory as compared with the standard implementation, and longer pedicle polyaxial screws were implanted in a more inclined angle at the fracture level. These intermediate polyaixal screws can help stabilize the anterior and middle columns of the fractured vertebrae, which enhance the interface strength and contribute to stability of the SSIF [22, 23]. Therefore, a retrospective study was conducted to compare the feasibility, safety and efficacy of SSIF with inclined-angle polyaxial screw (SSIF-IAP) with SSIF with straight-forward monoaxial screw (SSIFSFM) and LSIF with straight-forward monoaxial screw in the treatment of Denis type B TL fracture.

\section{Material And Methods}

\section{Patient population}

78 consecutive patients met the criteria for inclusion. 5 patients refused the surgical treatment, and 4 were lost to follow-up. 69 patients (47 males and 22 females), ranging from 22 to 54 years old (average 34.5 years) were enrolled in this study. Only patients with a single-level TL junction (T11-L2) fracture were included and received surgical management in our department between May 2011 and May 2015. Patients were divided into three groups:1. SSIF-IAP: SSIF with intermediate inclined-angle polyaxial screw at the fracture level; 2. SSIF-SFM: SSIF with intermediate straight-forward monoaxial screw at the fracture level; 3. LSIF: Long-segment internal fixation using two monoaxial pedicle screws above and below the fracture level (Figure 1).

The study was approved by the Ethics Committee of General Hospital of Central Theater Command and were in accordance with the Helsinki Declaration. Written informed consent was obtained from each 
patient..

\section{Inclusion and exclusion criteria}

The inclusion criteria were: Denis type B TL fracture, no unstable fracture dislocations, absence of obvious neurological impairment (Frankel grade $A$ and $B$ ), and from trauma to operation being less than one week. The exclusion criteria were: The fractured inferior endplate that was confirmed by computed tomography (CT) scans, pathologic fracture, osteoporotic fracture, bilateral pedicle fracture, previous spinal surgery history, other major organ system injuries, pregnancy.

\section{Surgical procedures}

After induction of general anesthesia with endotracheal intubation, each patient was placed in the prone position on a specialized operating frame that both shoulders and superior iliac spines were supported by gel pads to create hyperextension position of the spine and achieve postural reduction. In the SSIF-IAP group, after determination of the fracture level using fluoroscopy, a midline vertical skin incision was made to strip the erector spinae muscles bilaterally, and spinous processes and laminae were exposed. Four pedicle screws were bilaterally implanted into adjacent vertebrae above and below the fracture level. For the fractured vertebrae, semi- laminectomy or laminectomy was performed, and then a "L"-shaped chisel was inserted into the fractured vertebrae to restore the compressed and fractured vertebrae in the spinal canal. The superior endplate was injured, and the inferior endplate was intact in the Denis type B TL fracture, the starting point was $3 \mathrm{~mm}$ superior to the standard landmark, and the pedicle screws insertion was approximately 5 degrees inclined to the inferior endplate. Unilateral or bilateral pedicle screws were placed according to the integrity of the pedicle of the fractured vertebrae. The inclined-angle pedicle screws were purchased in the residual lower portion of the injured vertebral body. After all pedicle screws were attached, two rods connected pedicle screws on both sides using the rod placement system. The reduction and fixation were confirmed under fluoroscopy, and the incision was then irrigated and sutured. All operations were performed by the same surgery group. The procedures for LSIF and SSIF-SFM were described as previously [16]. Only one instrumentation technique without grafting fusion was utilized. If no spinal canal compression was observed before operation, pedicle screws can also be implanted percutaneously under $\mathrm{C}$-arm fluoroscopic guidance. The internal fixation stabilization system was supplied by Shandong Weigao Company of China.

All patients were routinely administered prophylactic antibiotics postoperatively for 48 hours, and the sterile dressing of incision was replaced every 2 days until the suture was removed. Patients were encouraged to start physical activities under the protection of brace, however, excessive and heavy activities were restricted up to 12 weeks after the operation. Following discharge from the hospital, 
patients were clinically and radiologically assessed at monthly intervals in the orthopedic outpatient clinic, with a mean follow-up of 24.01 months (range, 18-36 months).

\section{Clinical Assessment}

Radiographic evaluation consisted of sagittal Cobb's angle (SCA), anterior vertebral body height (AVBH), vertebral body index (VBI) and spinal canal encroachment (SCE). The SCA, AVBH and VBI were measured as previously described[17]. SCE was obtained from serial transverse CT scans by using Image $\mathrm{J}(\mathrm{NIH}$, Bethesda, MD) on admission and immediately after surgery, and it was used to evaluate the extent of the spinal canal decompression [24]. The correction losses of AVBH were calculated from the equation: postoperative AVBH (1-week) - postoperative AVBH (6-month or the latest)/ postoperative AVBH (1-week); The correction losses of VBI were calculated from the equation: postoperative VBI (1-week) postoperative $\mathrm{VBI}$ (6-month or the latest)/ postoperative VBI (1-week). All the data were analyzed by an independent observer who was not involved in the treatment of patients.

The clinical data from all included patients were obtained and assessed. We evaluated each patient by using Visual Analogue Scale (VAS) and Oswestry disability index (ODI) questionnaires, preoperatively and at each follow-up (1 week, 6 months and the latest follow-up). The VAS and ODI scores were recorded in the questionnaires at each follow-up in the orthopedic outpatient clinic. Statistical analyses

The Kruskal-Wallis test, Chi-square test and Mann-Whitney $\mathrm{U}$ test were performed to analyze the variables calculated by SPSS 17.0 software (SPSS Inc, Chicago, USA). Quantitative data were represented as the median (range) and a statistically significant difference with $P$ value $<0.05$. No alpha adjustment for multiple testing was performed.

\section{Results}

\section{Demographic characteristics}

Baseline demographic data, age, sex, body mass index (BMI), cause of injury, fracture site and neurological status in three groups were presented in Table 1. No significant differences were observed among three groups. The injured vertebrae segments were T11 in 7 cases, T12 in 14 cases, L1 in 31 cases, and L2 in 17 cases. The fractures were caused by traffic accidents in 25 cases, falling from height in 30 cases, and other accidents in 14 cases. 7 cases were in Frankel grade C, 22 cases in Frankel grade D and 40 cases in Frankel grade $\mathrm{E}$ (Table 1).

The average incision length was $10.4 \mathrm{~cm}(7.2-16.3 \mathrm{~cm})$. The mean blood loss was $110 \mathrm{ml}(30-450 \mathrm{ml})$ during operation. The average duration of operation was 122.4 mins (98-155 mins). The average hospital stay was 14.8 days (10-22 days). The most common reason for the delay was financial constraint. There were no significant differences between the SSIF-IAP group and the SSIF-SFM group with regard to incision length, mean blood loss, duration of operation and hospital stay. The average 
value of these parameters in the SSIF-IAP group and SSIF-SFM group were significantly lower than those in the LSIF group (Table 2).

\section{Radiologic outcomes}

Significant improvements from baseline in SCA, AVBH, VBI and SCE of the fractured vertebrae were observed among three groups $(P<0.05)$. There was no significant difference between the SSIF-IAP group and SSIF-SFM group with regard to SCA postoperatively $(P>0.05)$ however, SCA in the LSIF group was significantly more improved than those in the SSIF-SF group at 6 months $(P<0.05)$. Improvements in AVBH and VBI were not in agreement with SCA postoperatively. The AVBH in the SSIF-IAP group and LSIF group were significantly more improved than those in the SSIF-SFM group at 6 months and the latest follow-ups $(P<0.05)$. The VBI in the SSIF-IAP group and LSIF group were also significantly more improved than those in the SSIF-SFM group at 6 months and the latest follow-ups $(P<0.05)$. There were no significant differences among three groups postoperatively with regard to $\operatorname{SCE}(P>0.05)$ (Table 3$)$.

Similar to the results mentioned above, there were no significant differences among three groups with regard to correction losses of SCA $(P>0.05)$, however, the correction losses of AVBH in the SSIF-IAP group and LSIF group were significantly lower than those in the SSIF-SF group $(P<0.05)$; the correction losses of VBI in the SSIF-IAP group and LSIF group were also significantly lower than those in the SSIF-SF group $(P<0.05)$ (Table 4).

\section{Clinical outcomes}

The VAS scores following surgery improved in three groups. There were no significant differences among three groups with regard to the VAS scores at the pre-operation and 1-week follow-ups among three groups $(P>0.05)$, however, the VAS scores in the SSIF-IA group and SSIF-SF group were significantly lower than those in the LSIF group at the 6 months and the latest follow-ups $(P<0.05)$. The ODI scores following surgery also improved in three groups. Similarly, there were no significant differences among three groups with regard to the ODI scores at the pre-operation and 1-week follow-ups among three groups $(P>0.05)$, however, the ODI scores in the SSIF-IA group and SSIF-SF group were significantly lower than those in the LSIF group at the 6 months and the latest follow-ups $(P<0.05)$ (Table 5).

\section{Complications}

No major complications, such as nerve injury, wound infections, non-fusion etc., occurred among three groups postoperatively. One case of screw breakage at the 6-month follow-up, with a $4.16 \%$ failure rate in the SSIF-SFM group, and one case of screw loosing at the 1-year follow-up, with a $5.26 \%$ failure rate in 
the LSIF group, however, there were no difference between the two groups concerning failure rate $(\mathrm{P}=$ 0.87). Both patients underwent conservative treatment until solid bony fusion of the superior vertebrae was observed, and then the implant was removed. All patients with incomplete neurological impairment in three groups improved at least one grade according to Frankel performance scale. Functional recovery (Frankel Grades D or E) was observed in all patients postoperatively.

\section{Representative cases}

Representative case who underwent operation via inclined-angle screw placement was illustrated in Figure 2.

\section{Discussion}

Although the conservative management was thought to be the optimal treatment of TL junction fracture without serious neurological impairment, it is often accompanied by discomfort and limited mobility. Surgical intervention is therefore preferred in patients with TL junction fracture, because it can maintain the reduction, prevent further deformity and neurologic deterioration, and improve mobilization. Especially for young patients, surgical intervention may have advantageous effects for the recovery of spine sagittal alignment in the long run. The selection of the surgical approach in the treatment of TL junction fracture is dependent on many variables, such as bone intensity, kyphotic deformity, spinal canal encroachment etc. Either anterior, posterior approach or combined approaches can be applied for the stabilization of the unstable spine. Studies have shown that anterior instrumentation with bone graft can achieve reliable internal fixation, but it requires a more invasive approach that is associated with complications and prolonged postoperative recovery $[8,25]$. Alternative intervention was considered prior to this more invasive approach. Isolated posterior approach can also be applied to maintain the stability of the spinal column. LSIF via posterior approach can provide enough rigidity and stiffness of the spine, however, it might decrease spinal range of motion and increase the incidence of ASD. Therefore, other improved alternatives have been lately developed to take advantage of its benefits and minimize its adverse effects.

Superior biomechanical stability was found in SSIF with the addition of pedicle fixation at the fracture level without sacrificing the benefits of SSIF. Studies have shown that SSIF with intermediate screws insertion could significantly improve the biomechanical stability and construct stiffness as compared with SSIF $[11,26]$. Moreover, clinical research has found that the preservation of the fractured vertebrae height obtained in SSIF with intermediate screws was equivalent to that in LSIF [18]. Secondly, the use of intermediate screws at the fracture level can optimize load on instrumentation and reduce the risk of broken screws or rods. Buckling distortion of rod was more evident within the four-screw fixation construct than that within the six-screw fixation construct[27]. This is due to that the rod of the four-screw 
fixation construct spans a longer distance between two screws as compared with six-screw fixation construct, tension strains at each level of the four-screw fixation construct were significantly higher than that at each level of the six-screw fixation construct [28, 29]. Although traditional SSIF with intermediate screws theoretically corrects kyphotic deformities, however, the present instrumentation is not able to provide adequate support to the injured anterior spine column for unstable TL fracture in practice.

We then developed a new strategy for SSIF with inclined-angle intermediate polyaxial screws. There are some advantages as following: Firstly, this inclined-angle screw placement increases the length of pedicle screws, so it can provide greater construct stiffness and increase the pullout force. Denis type B fracture is a special categorized fracture, in which the superior endplate is mainly involved, while the inferior endplate and the lower portion of the injured vertebral body usually escapes from the injury site[30]. Therefore, the residual vertebral body and the caudal disc are preserved and they are able to tolerate anterior column reconstruction. The pedicle screws in the SSIF-IAP group were inserted into the lower residual portion of the injured vertebral body, which would contribute to the pullout strength. In addition, the "eggshell" deformity often occurred and the injured vertebrae can't provide enough construct stiffness during the healing process of fracture[31]. The potential reason for the "eggshell" effect is that the vertebral height is fully restored by the internal fixation device, but the compressed bone trabeculae are not restorable, which results in a defect in the injured vertebral body [32].To prevent this, several techniques have been developed to augment the anterior column in the unstable fractures, such as polymethylmethacrylate (PMMA) injection, however, injection of PMMA into the fractured vertebral body may often lead to cement extrusion, particularly when the posterior longitudinal ligament is torn[33]. Intermediate screws in the SSIF-SFM group are paralleled with the superior endplate, and the end portion of screws in the eggshell-like cavity can't provide additional interface strength. However, Intermediate screws in the SSIF-IAP group can escape from this cavity, and contribute to the construct stiffness. It might minimize the negative effects caused by the "eggshell" deformity, and promote fracture healing by increasing structural stability. However, no data are available to support this assumption that needed to be verified by further biomechanical study. During the follow-up period of over 2 years, none of patients in the SSIF-IAP group exhibited loosening or shifting of the intermediate screws at the fracture level. We believed that the main reason for this difference might be due to that screw-to-bone interface strength was improved by the increased angulation of the screws, and the anterior and middle spinal columns were immediately strengthened by these inclined-angle polyaxial screws. It suggests that inclined-angle screws at the fracture level can improve construct stiffness and protect the fractured vertebral body from anterior loads.

This retrospective study evaluated the radiological outcomes of 69 patients with TL fracture who were treated with three different internal fixations. As it was observed in the current study, SSIF with intermediate inclined-angle screws provided better postoperative correction and maintenance than SSIF 
with intermediate straight-forward screws. There was no significant difference among three groups with regard to SCA. However, changes of AVBH and VBI were not in agreement with SCA postoperatively. The initial correction of AVBH and VBI in the SSIF-IAP group were better than those in the SSIF-SF group. Moreover, the correction losses of the AVBH and VBI in the SSIF-IAP group were also significantly lower than those in the SSIF-SFM group at the 6 months and the latest follow-ups. Although AVBH, VBI and SCA are important parameters for the restoration evaluation of fractured vertebrae, however, sometimes they don't go hand in hand[34]. We attributed minor changes of SCA to the fact that intermediate inclinedangle screws might restore the height of fractured vertebrae more effectively as compared with the correction of kyphotic angle, which was Similar to the previous study [34].No statistical difference of SCE among three groups is due to no significant correlation between canal narrowing and neurological encroachment, which is dependent on the injury of spinal cord occurs at the time of trauma[35]. Only patients with minor neurological impairment (Frankel grade C, D and E) were included in our study, and all of them gradually recovered thereafter. Although the correction and maintenance of the fractured vertebral body was the best in the LSIF group, however, from a statistical point of view, the statistical difference for the correction losses between the SSIF-IAP and LSIF was not significant. Our data supported that the SSIF-IAP was comparable to LSIF, and it also can offer improved fixation and better correction than SSIF-SFM in the treatment of TL junction fractures.

Similar to the previous study, the values of all considered parameters (incision length, blood loss, surgical duration and hospital stay) in the LSIF group were the highest among three groups, however, no significant differences were observed for these parameters between the SSIF-IAP group and SSIF-SFM group. Moreover, significant improvements of functional outcomes (VAS back pain and ODI) were obtained in the SSIF-IAP group and SSIF-SFM group as compared with that in the LSIF group at the 6month and the last follow-up. Favorable surgical outcomes can be defined by $15 \%$ improvement in ODI scores[36], and our data were consistent with this criteria. In addition, it was found that ODI score is associated with VAS and SF-36[37]. The ODI changes might be explained by the corresponding VAS changes in our study. These results suggested that intermediate inclined-angle screws insertion at the fracture level didn't increase the operation duration and the blood loss as compared with the traditional straight-forward screws insertion.

Alvine et al. [38]reported that $39 \%$ screw breakage was found and $23 \%$ reoperation was performed. McLain et al. [39] have shown that instrumentation failure incidence was more than 50 percent. In our series, instrumentation failure occurrence was lower than that reported in these studies, one case of screw breakage in the SSIP-SFM group and one case of screw loosing in the LSIP group (instrumentation failure rate $=2.90 \%$ ). One screw breakage above the fracture level was observed at the 6-month follow-up in a 28 -years old man (instrumentation failure rate $=4.16 \%$ ). We attributed the reason for this instrumentation failure to the increased stress on the pedicle screw. This man had a history of heavy 
work without brace protection postoperatively. One screw loosing occurred at the 1-year follow in a 72years old woman (instrumentation failure rate $=5.26 \%$ ). This patient was diagnosed as osteoporosis preoperatively, however, she didn't follow the doctor's advice and take medicine against osteoporosis regularly during the follow-up.

There are still several limitations to this study. First, underlying factors such as, the bone density, degree of disc degeneration, and vertebral size, are variable. These confounding factors were offset by investigating three internal fixation strategies in the same specimen. Again, these clinical observations were based on data from relatively healthy strong bones (average 34.5 years), and a different picture might emerge in osteoporotic bones. In addition, this study evaluated short-term and small-population clinical outcomes, and findings may be biased, a long-term and large-scale prospective study should be performed to accurately evaluate the feasibility of this technique. Lastly, the speculation in this study was based on clinical observation, future bio-mechanical research needed to be conducted to support this application.

In conclusion, the SSIF-IAP can exert greater strength on the fractured vertebrae and effectively maintain the height of fractured vertebrae as compared with SSIF-SF; Compared with the LSIF, the SSIF-IAP can minimize the number of fused levels, and promote rapid relief of lumbar back pain and early rehabilitation. We recommended that SSIF with inclined-angle screws was effective and reliable operative technique for patients with Denis type B TL fracture.

\section{Abbreviations}

SSIF: Short-segment internal fixation; SSIF-IAP: Short-segment internal fixation with inclined-angle polyaxial screw; SSIF-SFM: Short-segment internal fixation with straight-forward monoaxial screw; LSIF: Long-segment internal fixation; TL: thoracolumbar; SCA: Sagittal Cobb's angle; AVBH: Anterior vertebral body height; VBI: Vertebral body index; SCE: Spinal canal encroachment; VAS: Visual Analogue Scale; ODI: Oswestry disability index.

\section{Declarations}

\section{Acknowledgments:}

Not applicable.

\section{Research registration number:}


Not applicable.

\section{Disclaimer:}

On behalf of all authors, the corresponding author states that there is no conflict of interest.

\section{Author contributions:}

FX and HK have designed the study, and performed the operations. JW have collected the data. CX have analyzed the data and made the statistics. BH have written the manuscript. All authors read and confirmed the final manuscript.

\section{Funding:}

This work is supported by the Natural Science Foundation of China (Grantno:81401802) and the Natural Science Foundation of Hubei Province (Grantno:2018CFB487) and the funds from National Health and Family Planning Commission of Wuhan Municipality and China Postdoctoral Science Foundation (Grant no:2016M593043).

\section{Availability of data and materials:}

The datasets for this study are available from the corresponding author on reasonable request.

\section{Ethics approval and consent to participate:}

Written informed consent was obtained from each patient included in the study. This study was according to the Helsinki Declaration and was approved by the local ethics committee.

\section{Consent for publication:}

Not applicable. 
No competing interests were declared.

\section{References}

1. P Leucht KF, G Muhr, EJ Mueller: Epidemiology of traumatic spine fractures. Injury 2009, 40(2):166172.

2. Tian Y ZY, Yin B, Zhang F, Liu B, Chen W, Zhang Y: Age- and gender-specific clinical characteristics of acute adult spine fractures in China. Int Orthop 2016, 40(2):347-353.

3. Siebenga J LV, Segers MJ, Elzinga MJ, Bakker FC, Haarman HJ, Rommens PM, ten Duis HJ, Patka P: Treatment of traumatic thoracolumbar spine fractures: a multicenter prospective randomized study of operative versus nonsurgical treatment. Spine (Phila Pa 1976) 2006, 31(25):2881-2890.

4. SD G: Neurologic deterioration in patients with thoracic and lumbar fractures after admission to the hospital. Spine (Phila Pa 1976) 1994, 19(15):1723-1725.

5. W D: Innere Fixation von Brust- und Lendenwirbelfrakturen. Aktuel Probl Chir Orthop 1984, 28:1-125.

6. Roy-Camille R SG, Maze1 C: Internal fixation of the lumbar spine with pedicle screw plating. Clin Orthop 1986, 203:7-17.

7. Shono Y MP, Cunningham BW: Experimental study of thoracolumbar burst fractures. A radiographic and biomechanical analysis of anterior and posterior instrumentation systems. Spine (Phila Pa 1976) 1994, 19(15):1711-1722.

8. Sasso RC RK, Hanson D, Reilly T, McGuire RA Jr, Best NM: Unstable thoracolumbar burst fractures: anterior-only versus short-segment posterior fixation. J Spinal Disord Tech 2006, 19(4):242-248.

9. Sasso RC BN, Reilly TM, McGuire RA Jr: Anterior-only stabilization of three-column thoracolumbar injuries. J Spinal Disord Tech 2005, 18 Suppl:S7-14.

10. Okten AI GY, Ozsoy KM, Ates T, Menekse G, Aslan A, Cetinalp E, Guzel A: Results of treatment of unstable thoracolumbar burst fractures using pedicle instrumentation with and without fracture-level screws. Acta Neurochir (Wien) 2015, 157(5):831-836.

11. Mahar A KC, Wedemeyer M, Mitsunaga L, Odell T, Johnson B, Garfin S: Short-segment fixation of lumbar burst fractures using pedicle fixation at the level of the fracture. Spine (Phila Pa 1976) 2007, 32(14):1503-1507.

12. McLain RF SE, Benson DR: Early failure of short-segment pedicle instrumentation for thoracolumbar fractures. A preliminary report. J Bone Joint Surg Am 1993, 75(2):162-167.

13. Nagata H SM, Transfeldt EE, Lewis JL: The effects of immobilization of long segments of the spine on the adjacent and distal facet force and lumbosacral motion. Spine (Phila Pa 1976) 1993, 18(16):2471-2479.

14. Dekutoski MB SM, Ogilvie JW, Olsewski JM, Wallace LJ, Lewis JL: Comparison of in vivo and in vitro adjacent segment motion after lumbar fusion. Spine (Phila Pa 1976) 1994, 19(15):1745-1751.

15. Park P GH, Gala VC, Hoff JT, McGillicuddy JE: Adjacent segment disease after lumbar or lumbosacral fusion: review of the literature. Spine (Phila Pa 1976) 2004, 29(17):1938-1944. 
16. Wang H ZY, Li C, Liu J, Xiang L: Comparison of Open Versus Percutaneous Pedicle Screw Fixation Using the Sextant System in the Treatment of Traumatic Thoracolumbar Fractures. Clin Spine Surg 2017, 30(3):E239-E246.

17. Li K LZ, Ren X, Xu H, Zhang W, Luo D, Ma J: Effect of the percutaneous pedicle screw fixation at the fractured vertebra on the treatment of thoracolumbar fractures. Int Orthop 2016, 40(6):1103-1110.

18. Guven O KB, Bezer M, Aydin N, Nalbantoglu U: The use of screw at the fracture level in the treatment of thoracolumbar burst fractures. J Spinal Disord Tech 2009, 22(6):417-421.

19. Tezeren G KI: Posterior fixation of thoracolumbar burst fracture: short-segment pedicle fixation versus long-segment instrumentation. J Spinal Disord Tech 2005, 18(6):485-488.

20. Kayanja M EK, Milks R, Lieberman IH: The mechanics of polymethylmethacrylate augmentation. Clin Orthop Relat Res 2006, 443(124-30).

21. Yang SC LP, Tu YK: Pullout evaluation of sawbone experiment in different types of pedicle screws combined with bone cement augmentation for severe osteoporotic spine. Acta Bioeng Biomech 2018, 20(2):55-64.

22. Feng ZZ CY, Jiang C, Jiang XX: Short-term outcome of bilateral decompression via a unilateral paramedian approach for transforaminal lumbar interbody fusion with unilateral pedicle screw fixation. Orthopedics 2011, 34(5):364.

23. Xiao YX CQ, Li FC: Unilateral transforaminal lumbar interbody fusion: a review of the technique, indications and graft materials. J Int Med Res 2009, 37(3):908-917.

24. Boisclair D M-TJ, Parent S, Petit $Y$ : Compressive loading of the spine may affect the spinal canal encroachment of burst fractures. J Spinal Disord Tech 2013, 26(6):342-346.

25. Sasso RC BN, Reilly TM, McGuire RA Jr: Anterior-only stabilization of three-column thoracolumbar injuries. J Spinal Disord Tech 2005, 18 Suppl:18 Suppl.

26. Dick JC JM, Zdeblick TA, Kunz DN, Horton WC: A biomechanical comparison evaluating the use of intermediate screws and cross-linkage in lumbar pedicle fixation. J Spinal Disord Tech 1994, 7(5):402-407.

27. Norton RP ME, Kaimrajh DN, Eismont FJ, Latta LL, Williams SK: Biomechanical analysis of fourversus six-screw constructs for short-segment pedicle screw and rod instrumentation of unstable thoracolumbar fractures. Spine J 2014, 14(8):1734-1739.

28. TA Z: A prospective, randomized study of lumbar fusion. Spine (Phila Pa 1976) 1993, 18:983-991.

29. Schwab FJ ND, Mahmud F, Michelsen CB: Effects of spinal instrumentation on fusion of the lumbosacral spine. Spine (Phila Pa 1976) 1995, 20(18):2023-2028.

30. F D: Spinal instability as defined by the three-column spine concept in acute spinal trauma. Clin Orthop Relat Res 1984, 189:65-76.

31. Xu BS TT, Yang HL: Long-term results of thoracolumbar and lumbar burst fractures after shortsegment pedicle instrumentation, with special reference to implant failure and correction loss. Orthop Surg 2009, 1(2):85-93. 
32. Dengwei He LW, Xiaoyong Sheng,Qinqin Xiao,Ye Zhu,Weiyang Yu,Feijun Liu,Kejun Zhu: Internal fixation with percutaneous kyphoplasty compared with simple percutaneous kyphoplasty for thoracolumbar burst fractures in elderly patients: a prospective randomized controlled trial. Eur Spine $J$ 2013, 22(10):2256-2263.

33. Cho DY LW, Sheu PC: Treatment of thoracolumbar burst fractures with polymethyl methacrylate vertebroplasty and short-segment pedicle screw fixation. Neurosurgery 2003, 53(6):1354-1360.

34. Tian JW WL, Xia T, Liu CY, Zhao QH, Dong SH: Posterior short-segmental fixation combined with intermediate screws vs conventional intersegmental fixation for monosegmental thoracolumbar fractures. Orthopedics 2011, 34(8):e389-396.

35. Waters RL AR, Yakura JS, Sie I: Effect of surgery on motor recovery following traumatic spinal cord injury. Spinal Cord 1996, 35:266-274.

36. R. Casal-Moro MC-M, M. Hernández-Blanco, J. A. Bravo-Ricoy, F. J. Jorge-Barreiro: Long-term outcome after microendoscopic diskectomy for lumbar disk herniation: a prospective clinical study with a 5-year follow-up. NEUROSURGERY 2011, 68(6):1568-1575.

37. H. Liu HT, Z. Luo: Validation of the simplified Chinese version of the Oswestry Disability Index. Spine (Phila Pa 1976) 2009, 34(11):1211-1216.

38. Alvine GF SJ, Asher MA, Burton DC: J Spinal Disord Tech. Treatment of thoracolumbar burst fractures with variable screw placement or Isola instrumentation and arthrodesis: case series and literature review 2004, 17(4):251-264.

39. McLain RF SE, Benson DR: Early failure of short-segment pedicle instrumentation of thoracolumbar fractures. A preliminary report. J Bone Joint Surg Am 1993, 75(2):162-167.

\section{Tables}

Please see the supplementary files to access the tables.

\section{Figures}




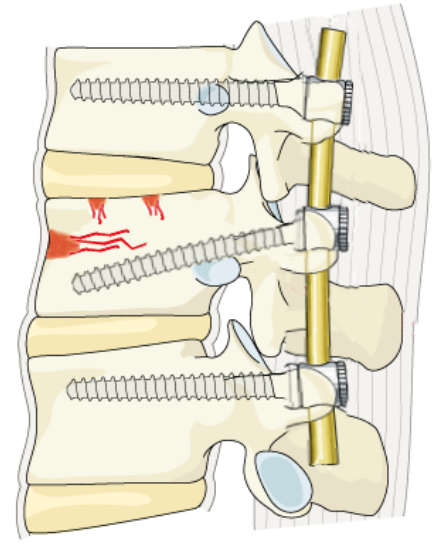

A

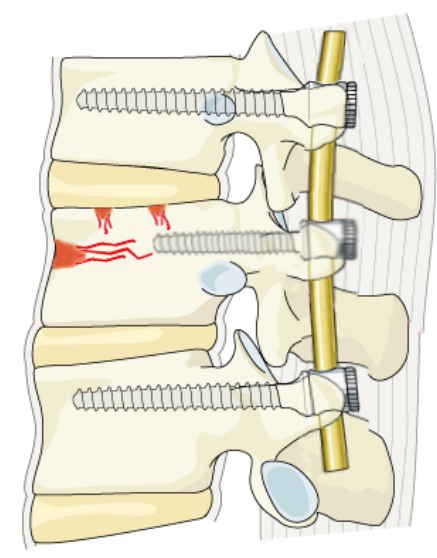

B

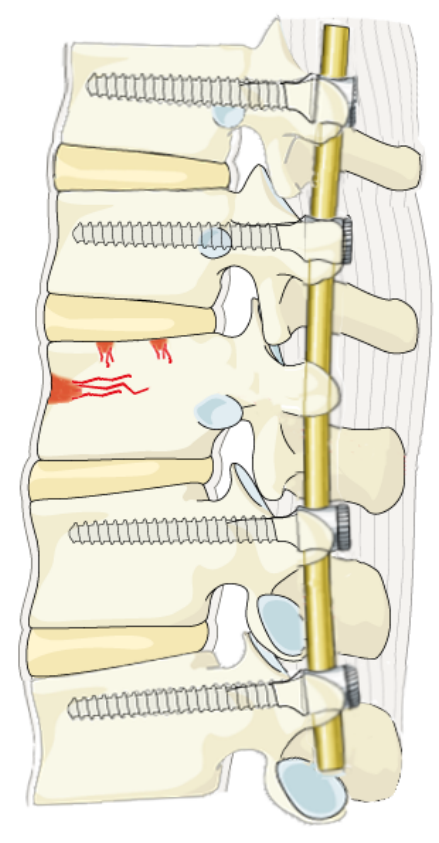

C

\section{Figure 1}

The illustration of three different fixation on the treatment of Denis type B TL fracture. A: Inclined-angle screws were inserted at the fracture via the posterior approach along with SSIF; B: Straight-forward screws were inserted at the fracture via the posterior approach along with SSIF; C: Two pedicle screws above and below the fracture level were inserted via the posterior approach along with LSIF. TL: thoracolumbar; SSIF: short-segment internal fixation; LSIF: Long-segment internal fixation. 

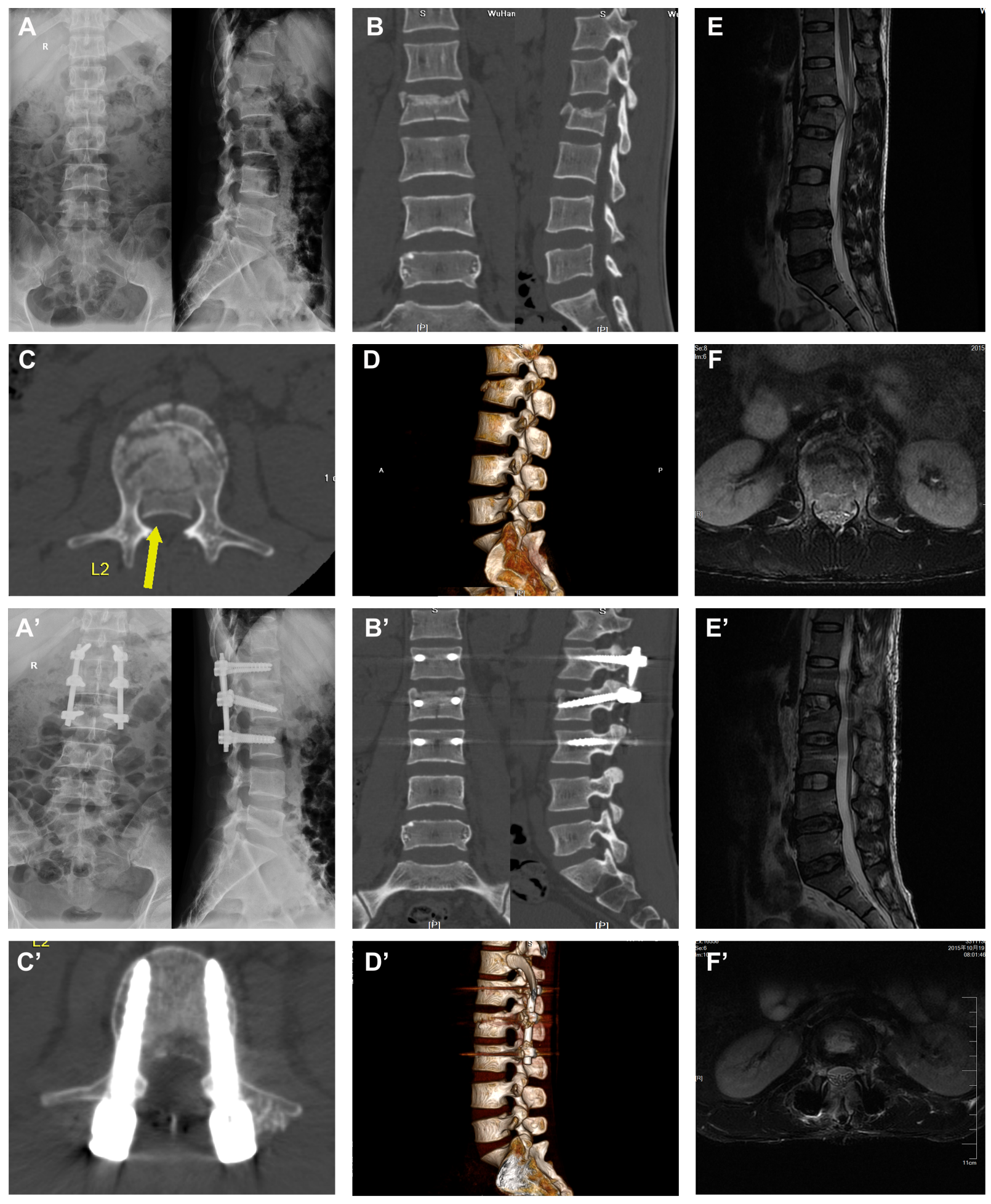

\section{Figure 2}

X-Ray, CT and MRI of a case with Denis type B TL fracture treated by SSIF-IA system. A and A': Anteroposterior and lateral X-Ray demonstrating the L2 compression fracture before and after operation; $B$ and $B$ ': Lateral spiral CT showing the $L 2$ fracture with injured superior endplate before and after operation; $C$ and $C^{\prime}$ : Axial spiral CT showing the spinal canal encroachment by fragments of fracture vertebra before and after operation; D and D': The 3D reconstruction of lumbar spine with L2 compression 
fracture before and after operation; $E$ and E': Sagittal MRI confirming the L2 compression fracture with vertebra edema before and after operation; $F$ and $F^{\prime}$ : Axial MRI confirming the spinal canal encroachment by fragments of fracture vertebra along with posterior elements of the vertebrae before and after operation. CT: computer tomography; MRI: magnetic resonance imaging; 3D:3-dimension.

\section{Supplementary Files}

This is a list of supplementary files associated with this preprint. Click to download.

- ModifiedTable3.jpg

- ModifiedTable4.jpg

- ModifiedTable1.jpg

- ModifiedTable2.jpg

- ModifiedTable5.jpg 